\title{
Ultra-Iow Noise Differential Pre-amplifier Design and Its Noise Floor Measurement
}

\author{
Xiu-hui $\mathrm{Ni}^{1},{ }, \mathrm{Yi}$ Zheng ${ }^{2,}$ and $\mathrm{Yan} \mathrm{Xu}^{3}$ \\ 1, 2, 3 Institution of Oceanographic Instrumentation, Shandong Academy of Sciences, \\ Qingdao 266001, China
}

Keywords: Low noise, Preamplifier, Noise measurement.

\begin{abstract}
This paper describes a differential amplifier which is based on the discrete/op-amp combo design, the noise performance test method and the test results are also given. Experiments shows that an ultra-low noise amplifier are achieved, the Bipolar input amplifier's input equivalent noise density is less than $2 \mathrm{nv} / \mathrm{rt}-\mathrm{Hz}$, the BIFET input amplifier's input equivalent noise density is less than $5 \mathrm{nv} / \mathrm{rt}-\mathrm{Hz}$. The combo design scheme presents high low-noise performance which matches traditional complex discrete component design but exceeds it with much more simplicity and feasiblility.
\end{abstract}

\section{Introduction}

Attenuation characteristics of transmission in marine acoustic channel [1] cause higher demand for receiving sensitivity of electronic systems. With the increasing requirement for weaker signal reception in marine acoustic instruments, low noise preamplifier design and implementation become one of the important topics of current research. The key element of improve receiving sensitivity is to decrease the receiver's noise. The preamplifier[2] is the key component, which could decide the noise of the receiver.

The OP Amp is widely used in pre-amplifier, but its noise is not low enough and can't reach some specific marine instrument project application level. Some low noise transistor[3] have good noise character, but most of discrete component schemes designed using transistors or JFETs and discrete components are more difficult to achieve because of its complexity and extra pair works.

\section{The Design of Low Noise Preamplifier}

In this paper we describes a differential amplifier which is based on the discrete/op-amp combo design. The design achieves very low noise floor which beyond the operational Amplifier scheme meanwhile eliminating the complexity of pair work of discrete component scheme. The circuit consists of low noise output amplifier, input buffer with paired bipolar transistor $\mathrm{J} 1, \mathrm{~J} 2$ and transistor bias voltage source. It is shown in figure 1.Using the bipolar transistor $\mathrm{J} 1, \mathrm{~J} 2$ as buffer amplifier,U1A has even large open loop gain which makes this circuit performance getting close to an ideal operational amplifier. The amplifier's output is fed back to input. This deep negative feedback mechanism could make compensation for consistency of transistors automatically [4]. 


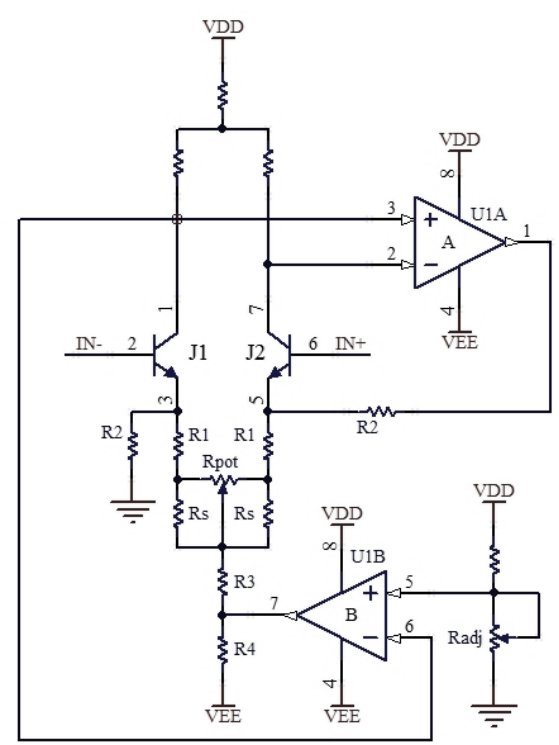

Figure1. Low Noise Differential Amplifier Scheme

The gain is determined by the proportion of feedback network:

$$
\mathrm{Av}=\frac{\mathrm{R} 2}{\mathrm{R} 1}
$$

The circuit could achieve amplify function with very low noise floor. The temperature stability of discrete component is also improved by the negative feedback[5]. Some key points of the design are shown in following:

\section{Input Stage Noise is the Main Noise Source of the Preamplifier}

By using the ultra-low noise discrete component such as bipolar transistor and field-effect tube as input buffer firstly, the circuit noise is determined basically by the performance of discrete component and decrease the influence of U1Anoise to the circuit.

Low noise discrete elements include bipolar transistor and field-effect tube(BIFET) generally have lower noise floor than the off-the-shelf Op AMPs [6]. Smaller impedance eg. $1 \mathrm{~K} \Omega$ (geophone preamplifier) application should select bipolar transistor as input stage to get lower input voltage noise. The noise of matched bipolar transistor could be lower than $2 \mathrm{nv} / \mathrm{rt}-\mathrm{Hz}$. It is shown in table 1:

Table 1. The noise characteristic of paired NPN transistor

\begin{tabular}{|c|c|c|c|}
\hline \multirow{2}{*}{ Type } & \multicolumn{3}{|c|}{ main characteristics } \\
\cline { 2 - 4 } & $\begin{array}{c}\text { Voltage Noise } \\
\mathrm{nV} / \mathrm{rt}-\mathrm{Hz}\end{array}$ & $\begin{array}{c}\text { Current Gain } \\
\mathrm{hFE}\end{array}$ & $\begin{array}{c}\text { Vos T Drift } \\
\mu \mathrm{V} /{ }^{\circ} \mathrm{C}\end{array}$ \\
\hline SSM2210 & 1.0 & $0.5 \%$ & 0.03 \\
\hline MAT12 & 1.0 & $0.5 \%$ & 0.03 \\
\hline LM394 & 1.8 & $2.0 \%$ & 0.10 \\
\hline THAT304 & 0.8 & $4.0 \%$ & 0.50 \\
\hline
\end{tabular}

BIFET has ultra-low current noise[7], which is more suitable for high impedance circuit, e.g. Hydrophone amplifier. The relative devices are shown in table 2: 
Table 2. The characteristics relative paired FET

\begin{tabular}{|c|c|c|c|}
\hline \multirow{2}{*}{ Type } & \multicolumn{3}{|c|}{ main characteristics } \\
\cline { 2 - 4 } & $\begin{array}{c}\text { Wideband Noise } \\
\mathrm{nV} / \mathrm{rt}-\mathrm{Hz} \\
@ 1 \mathrm{kHz}\end{array}$ & $\begin{array}{c}\text { Input } \\
\text { Cutoff Voltage } \\
\text { (Max) }\end{array}$ & $\begin{array}{c}\text { Input } \\
\text { Current Ratio } \\
\text { (Min) }\end{array}$ \\
\hline LSK389A & 0.9 & 20 & 0.90 \\
\hline 2N5564 & 2.0 & 5 & 0.95 \\
\hline 2SJ109 & 1.1 & 20 & 0.90 \\
\hline
\end{tabular}

\section{Static DC Operation Point and Base Current are Set by the DC-servo}

For the bipolar transistor, the emitter-base voltage noise is a function of temperature $\mathrm{T}$ and collector current Ic:

$$
e_{n}=k T \sqrt{\frac{2}{q I_{c}}} \text { Volts } / \sqrt{H z}
$$

In this equation, $\mathrm{k}$ is Boltzmann constant, $\mathrm{q}$ is the electron charge.

Actually, the circuit noise wouldn't decrease when Ic exceeds certain limit[10] because of the parasitic transistor noise. So for the finite impedance source $r_{s}$, the base current noise should add to voltage noise as orthogonal component, the total input equivalent noise could be represented by following equation:

$$
e_{N}=\sqrt{e_{n}^{2}+\frac{2 q \times I c \times r_{s}^{2}}{h_{F E}}+4 k T r_{s}}
$$

In this equation, $h_{F E}$ is the amplification factor of transistor, if $\mathrm{e}_{N}=0$ then we could get ideal collector current for lowest noise Characteristics.

$$
I_{c}(o p t)=\frac{k T \sqrt{h_{F E}}}{q r_{s}}
$$

A DC-servo is designed in the preamplifier, the source terminal voltage of transistor $\mathrm{J} 1, \mathrm{~J} 2$ is stabilized on the setting operation point due to the virtual short of the positive and the negative terminal of the U1A Op AMP, and the setting operation point voltage could be adjusted by $\mathrm{R}_{a d j}$ meanwhile the transistor's base current is settle down. By adjusting $I_{C}$ to the value calculated by the equation 4, the lowest total circuit noise could be achieved.

\section{Method to Decrease the Effect of Offset Noise}

The offset current of input buffer could generate huge system noise if not deployed correctly. One of the best methods to decrease the effect of offset noise is to add attenuation resistance in offset current path. In figure 1, R3 could decrease the noise(with coefficient $1+\mathrm{GmR} 3$ ) and increase noise performance[9].

\section{The Method of Eliminating the Input Imbalance Voltage}

The mismatch of input discrete element is main reason of input imbalance voltage, for example the datasheet of LSK389 shows that the interference could change Idss more than 10\%[9], it could effect on VGS and inject imbalance voltage. This differential amplifier could make zero drift voltage $\mathrm{mV}$ in dozens on input short condition without voltage compensation.

It is fortunate that the imbalance voltage is caused by the unequaled offset current of discrete element, which could be adjusted for solution. Figure 1 shows a solution: set an offset adjusting circuit with Rpot and Rs to adjust the input current. It is easy to 
decrease the imbalance voltage to $\mathrm{uV}$ level through offset adjust by accuracy control of Rpot.

\section{Other Consideration}

It is better to improve the symmetry of differential circuit to decrease temperature drift error [11]. So the metal film resistance with lower temperature drift should be selected. It is also appropriate to use resistance in same batch for better matching.

The low noise linear power should be applied with appropriate filtering, otherwise the power noise of coupled amplifier circuit could decrease the amplifier noise index[13].

The preamplifier circuit should be shield by tinplate to shield the electromagnetic interference in air[14].

\section{The Scheme and Result of Noise Measurement}

This measurement is to measure the output noise voltage of the unit on input short condition. Due to the weak signal, the signal of device under test could be contaminated by electromagnetic interference easily. So the selection of test devices and test method are both very important.

\section{Test Devices}

There are generally three types of test devices: RMS meter, oscilloscope and spectrum analyzer. RMS meter could measure the AC signal RMS voltage of various waveforms, the oscilloscope could be used for the noise test. But they could be both interfered by power-line interference without electromagnetic shielding and get large errors. The spectrum analyzer could show the relationship between Power (Voltage) and Frequency, it shows spectral density $(\mathrm{nV} / \mathrm{rt}-\mathrm{Hz})$ and make intuitive sight on the distribution of noise in different frequency bands. The isolation of power-line interference noise energy could conquer the above test difficulties.

\section{Test Method}

Because the preamplifier circuit we design in this paper has ultra-low noise voltage, which is far less than inherent noise of general test device. So it is better to set the first pre amplifier for the noise voltage of DUT, and then use spectrum analyzer to test, the method is in figure 2 :

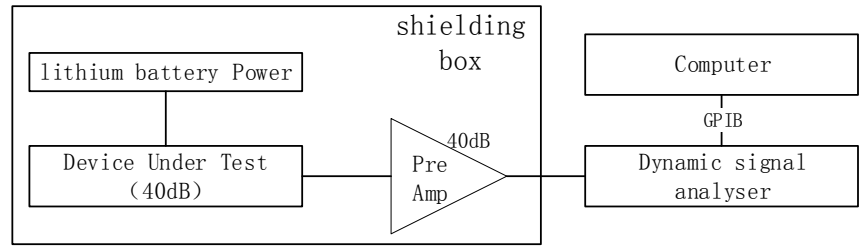

Figure2. Low Noise Test System

Put the DUT, lithium battery and low noise preamplifier into the shield box to make whole shielding. This setting could decrease the influence of power-line interference and electromagnetic noise. The dynamic signal analyzer [15] measure the output noise power of amplifier, and make communication with PC by GPIB interface.

\section{Test Result}

The test result as noise spectral density from dynamic signal analyzer is shown in figure 3 , in figure the spike in waveform is power-line interference. The dotted line below represents $20 \mathrm{uV}$ voltage. 


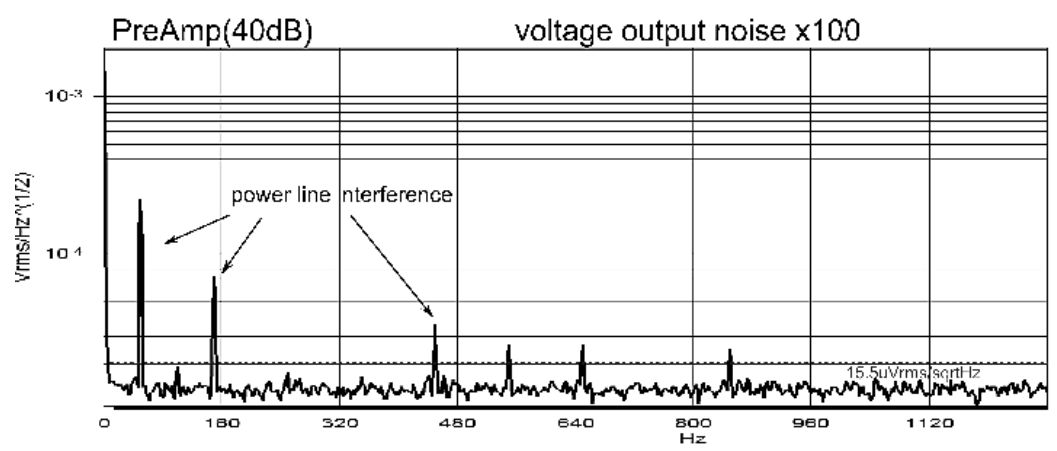

Figure3. The Noise Spectral Density from Dynamic Signal Analyzer

The related noise spectral density is $15.5 \mathrm{uV} / \mathrm{rt}-\mathrm{Hz}$ at $1 \mathrm{KHz}$. Because preamplifier has 40dBgain and pre amplifier also has 40dBgain, the input noise should be divided by 10000, the formula is in following:

$$
\mathrm{Ei}=\frac{15.5 \times 10^{-6}}{100 \times 100} V=1.55 \mathrm{nv} / \sqrt{\mathrm{Hz}}
$$

We measure 5 systems, each has 4 preamplifiers, $X, Y, Z$ are corresponding to 3 bipolar preamplifiers for low source impedance application, $\mathrm{P}$ is corresponding to 1 BIFET preamplifier for high source impedance application, the result of equivalent input noise voltage are shown in table 3. By repeating measurement, the test standard deviation is blow 0.5 .

Table 3. The result of input equivalent noise test for preamplifiers

\begin{tabular}{|c|c|c|c|c|}
\hline & $\mathrm{X}$ & $\mathrm{Y}$ & $\mathrm{Z}$ & $\mathrm{P}$ \\
\hline 1 & 1.28 & 1.23 & 1.26 & 4.7 \\
\hline 2 & 1.28 & 1.17 & 1.23 & 5,7 \\
\hline 3 & 1.22 & 1.33 & 1.18 & 5.1 \\
\hline 4 & 1.36 & 1.25 & 1.23 & 5.5 \\
\hline
\end{tabular}

\section{Conclusion}

In this paper we represent a differential amplifier which consist of a single double-amplifier chips, a single paired bipolar transistor (or BIFET) and a few resistors \& capacitors, it features ultra-low noise and the scheme could be used both in low and high source impedance applications. Several aspects of consideration were described in this paper and the key device type are also given. Through experiment it is proven that the low noise amplifier with ultra-low noise could be fulfilled.

The combo design scheme presents high low-noise performance which matches traditional complex discrete component designs [16][17]but exceeds it with much more simplicity and feasiblility.

This circuit can be used in weak signal detection such as the locked-in amplifier which needs high perform in noise characteristics. It has been used in several marine acoustics prototype instruments and has good performance.

\section{Acknowledgement}

This research was financially supported by the Natural Science Foundation of Shandong Province, China (ZR2013DM014) and the Key research and development Project of Shandong Province, China (2015GSF115018). 


\section{References}

[1] Wang Dezhao, Shang Erchang, Hydro acoustics, The Science Publishing Company, Beijing, 1981, pp. 21-25.

[2] C.D. Motchenbacher, You Zhongqi, Low noise electronic design, National Defense Industry, Beijing, 1977.

[3] Fang Zhihao, Tansistor low noise circuit, The Science Publishing Company, Beijing, 1984.

[4] Behzad Razavi, The design of analog CMOS integrated circuits, Xi'an Jiao Tong University Press, Xi'an, 2003, pp. 84-88.

[5] Sergio Franco, Liu Shutang, Zhu Maolin, Rong Meiyi, The cicuit design based on operational amplifier and analog integrated circuit, Xi'an Jiao Tong University Press, Xi'an, 2005, pp. 277-305.

[6] Zhang Yongjun, Several problems in the design of ultra low noise preamplifier, Petroleum Instrument, 1997, 11(3) 20-22.

[7] Yin Hui, The research and design of low noise amplifier based on BIFET, Journal of Jilin University,2009.

[8] Sergio Franco, Kang Baowei, Sun Wuyun, The design of field effect transistor circuit, People's Posts and Telecommunications Press, Beijing, 1988.

[9] Some Tips on Making a FETching Discrete Amplifier, Information on http://www.analog.com/library/analogDialogue/china/archives/47-10/discrete_amplifi er_cn.pdf

[10] An-222.Super Matched Bipolar Transistor Pair Sets New Standards for Drift and Noise, Information onhttp://www.ti.com/lit/an/snoa626b/snoa626b.pdf

[11] Wang Ling, Xu Yazhen, The design of amplifier circuit, The Science Publishing Company, Beijing, 2004, pp. 104.

[12] Zhou Shenghai, Guo Shuhong, The design of sensor preamplifier based on low noise operational preamplifier, Instrument technique and sensor, 2006, (9) 38-40.

[13] Gas Jinzhan, Weak Signal Detection, Tsinghua University Press, Beijing, 2011, pp.167-177.

[14] Henry, Wang Peiqing, Li Diyi, The restraint and attenuation technique of electronic system noise, Electronic Industry Publishing House, 2003, pp. 34-46.

[15] Stanford Research System, SR785 Operating Manual, Information onhttp://www.thinksrs.com/

[16] Chang Li, Design of ultra-low noise preamplifier,Chinese Journal of Electron Devices, 2008, 12(6) 1854-1856.

[17] Zhang Xiaofei,Design of JFET Low Noise Preamplifier,ChineseJournal OfEngineering Geophysics, 2009, 6(3) 348-351. 